\title{
Incentive Sensitization by Previous Amphetamine Exposure: Increased Cue-Triggered "Wanting" for Sucrose Reward
}

\author{
Cindy L. Wyvell and Kent C. Berridge \\ Department of Psychology, University of Michigan, Ann Arbor, Michigan 48109
}

\begin{abstract}
We reported previously that an amphetamine microinjection into the nucleus accumbens enables Pavlovian reward cues in a conditioned incentive paradigm to trigger excessive instrumental pursuit. Here we show that sensitization caused by previous amphetamine administration also causes reward cues to trigger excessive pursuit of their associated reward, even when sensitized rats are tested in a drug-free state. Rats learned to lever press for sucrose pellets, and they separately learned to associate sucrose pellets with Pavlovian cues (30 sec auditory cues). Amphetamine sensitization was induced by six daily injections of amphetamine (3 mg/kg, i.p.; controls received saline). Rats were tested for lever pressing under extinction conditions $10 \mathrm{~d}$ later, after a bilateral microinjection of intra-accumbens vehicle or amphetamine (5 $\mu \mathrm{g} / 0.5 \mu \mathrm{l}$ per side). Cue-triggered pursuit of sucrose reward was assessed by increases in pressing on the sucrose-associated lever during intermittent presentations of a free conditioned stimulus (CS+) sucrose cue. Sensitized rats pressed at normal levels during
\end{abstract}

In addicts, encounters with drug cues can trigger compulsive drug-seeking behavior even after long periods of drug abstinence (de Wit and Stewart, 1981; Jaffe et al., 1989; Goldstein, 1994; Topp et al., 1998; Mucha et al., 1999; Robbins et al., 1999). Many explanations have been offered for cue-triggered relapse, including conditioned withdrawal responses, conditioned positive reinforcement or drug-like effects, and conditioned stimulus-response habits (Robbins and Everitt, 1999; Siegel, 1999; Berke and Hyman, 2000; Ito et al., 2000). All of these processes may contribute to drug-taking behavior, but they may not provide a full explanation of why cue-triggered relapse can become compulsive. The incentive-sensitization theory of addiction suggests that neural sensitization in mesolimbic-related systems is needed to explain this process (Robinson and Berridge, 1993, 2000). It posits that sensitized neural circuits function to attribute incentive salience to reward-related stimuli, allowing reward cues to trigger excessive "wanting" for the reward whether or not it is correspondingly "liked" (Berridge and Robinson, 1998). When sensitized incentive salience is attributed to drug cues in addicts, it can trigger compulsive drug pursuit (Robinson and Berridge, 1993, 2000).

Received May 10, 2001; revised July 23, 2001; accepted July 25, 2001.

This research was supported by National Science Foundation Grant IBN 9604408 (K.C.B.) and by National Institute on Drug Abuse-National Research Service Award fellowship F31 DA0599901 (C.L.W.). We are grateful to Drs. J. Wayne Aldridge, Susana Peciña, and Terry E. Robinson for their helpful comments on an earlier version of this manuscript.

Correspondence should be addressed to Cindy Wyvell or Kent Berridge, University of Michigan, 525 East University Avenue, Ann Arbor, MI 48109-1109. E-mail: wyvell@umich.edu or berridge@umich.edu.

Copyright (C) 2001 Society for Neuroscience $\quad 0270-6474 / 01 / 217831-10 \$ 15.00 / 0$ baseline and during the CS-, but the CS+ triggered $100 \%$ greater increases in pressing from sensitized rats than from control rats after vehicle microinjection. Sensitization therefore enhanced the incentive salience attributed to the CS+ even when rats were tested while drug-free. For control rats, a microinjection of intra-accumbens amphetamine was needed to produce the same enhancement of cue-triggered reward "wanting." The amphetamine microinjection also interacted synergistically in sensitized rats to produce intrusive cuetriggered pursuit behaviors (e.g., investigatory sniffing) that interfered with goal-directed lever pressing. These results support the incentive-sensitization theory postulate that sensitization causes excessive cue-triggered "wanting" for an associated reward.

Key words: amphetamine; nucleus accumbens; mesolimbic; incentive sensitization; addiction; cue; Pavlovian; instrumental; dopamine; incentive salience; reward; conditioned reinforcement

Sensitization has been shown to enhance pursuit of even natural rewards. For example, sexual pursuit and mounting behavior of male rats toward females can be enhanced by previous morphine or amphetamine sensitization (Mitchell and Stewart, 1990b; Fiorino and Phillips, 1999a,b), possibly reflecting excessive enticement by sensitized sexual cues. Similarly, amphetamine sensitization enhanced the acquisition of cue-elicited approach to a cup for sucrose reinforcement (Harmer and Phillips, 1998, 1999a), perhaps reflecting excessive incentive salience attribution to the sucrose cue. Also, cocaine sensitization increased responding for a water cue in a conditioned reinforcement paradigm (Taylor and Horger, 1999), possibly reflecting sensitized incentive salience of the rewarding cue. The use of natural rewards in experiments allows study of the incentive-sensitization process relatively free from contamination by other consequences of drug use such as withdrawal.

However, these examples can alternatively be explained by increased primary or conditioned reinforcer impact after sensitization or by an increased learning of stimulus-reward or stimulus-response associations (Di Chiara, 1998; Robbins and Everitt, 1999; Berke and Hyman, 2000; Koob and Le Moal, 2001). What is needed is a way to distinguish these competing explanations.

Incentive-sensitization can be distinguished from other explanations by using a conditioned incentive paradigm based on Pavlovian-to-instrumental transfer (Dickinson et al., 2000; Wyvell and Berridge, 2000). In this paradigm, after separate Pavlovian and instrumental training sessions, cue-triggered wanting for sucrose reward is reflected by enhanced pressing on a sucroseassociated lever whenever reward cues are freely presented. Pri- 
mary reinforcement is avoided because the test is conducted under extinction conditions, so that sucrose is never received during testing. Similarly, conditioned response reinforcement is avoided because no contingency exists between the instrumental response and cue presentation. Finally, conditioned habits evoked by stimulus-response associations cannot facilitate responding because the instrumental response has never before been performed in the presence of the sucrose cue. Using this paradigm, Wyvell and Berridge (2000) showed that mesolimbic activation caused by an amphetamine microinjection into the nucleus accumbens selectively increased cue-triggered wanting for sucrose reward, without enhancing either response reinforcement or sucrose "liking." Here we demonstrate that previous drug sensitization can similarly endow reward cues with excessive incentive salience even when rats are tested while drug-free in a conditioned incentive paradigm.

\section{MATERIALS AND METHODS}

Subjects. Thirty female Sprague Dawley rats (born at the University of Michigan; 240-320 gm) were housed in pairs in plastic tub cages under a reverse $12 \mathrm{hr}$ light cycle (lights off at 10:00 A.M.). Rats were given $20-25$ gm of rat chow each day after the training or test session, and water was always provided ad libitum.

Test chambers. Each computerized operant chamber (Med Associates Inc., St. Albans, VT) contained a house light, sucrose cup (with photobeam entry detector), two levers, and speaker modules (clicker and tone). Video cameras were mounted beneath the transparent plastic floors to enable videotape recording of the rats' behavior. Sound attenuating boxes equipped with ventilation fans masked external noise.

Instrumental training. Rats were initially given two sessions of magazine training to shape them to eat from the sucrose cup. Twenty deliveries of a single $45 \mathrm{mg}$ sucrose pellet (Formula F; P.J. Noyes Co., Lancaster, PA) were given on a fixed time (FT), 1 min schedule of reinforcement. On days $3-17$, rats were trained daily in 30 min sessions to lever press for sucrose pellets, and the reinforcement requirement was incremented gradually to a variable interval (VI), $45 \mathrm{sec}$ schedule (i.e., FR-1, VI-5, VI-15, VI-30; VI-45). Presses on one lever in the operant chamber produced sucrose pellets, whereas presses on the other lever did not (and served only as a measure of sensorimotor arousal).

Pavlovian training. Both levers were absent from the chambers during Pavlovian conditioning on days 18-32 to prevent adventitious reinforcement of a lever pressing habit during the cue. Either the tone $(2.9 \mathrm{kHz}$, $0.5 \mathrm{sec}$ on/off continuous pulsing) or clicker stimuli were designated as the conditioned stimulus (CS+) (and the other was the CS-, counterbalanced across rats). During 14 daily sessions, rats received 10 pairings of the CS + (30 sec duration) with immediate delivery of three sucrose pellets [unconditioned stimulus (UCS)] on a variable time, 2 min schedule. To habituate any unconditioned responses to the CS-, the CS- (30 sec duration) was presented twice during the last three Pavlovian sessions (at the middle and end of each session) and was never followed by sucrose pellets (Dickinson et al., 2000). Approach to the sucrose cup was monitored, and difference scores were calculated to verify acquisition of the Pavlovian association (approaches during $30 \mathrm{sec} \mathrm{CS}+$ minus approaches during $30 \mathrm{sec}$ baseline immediately before the $\mathrm{CS}+$ ).

Drug sensitization. Rats were divided into two equal groups (sensitized versus control) that were matched for sucrose cup approach performance during the last day of Pavlovian conditioning. Sensitized rats received daily injections of amphetamine $\left(3 \mathrm{mg} \cdot \mathrm{ml}^{-1} \cdot \mathrm{kg}^{-1}\right.$, i.p. ) for $6 \mathrm{~d}$ (days 33-39), whereas control rats received saline vehicle injections (1 $\mathrm{ml} / \mathrm{kg}$ ). Immediately after each injection, the rats were placed into the operant chambers for $45 \mathrm{~min}$ (with the levers still absent). Locomotor activity was videotaped on the first and last day to confirm psychomotor sensitization. A $10 \mathrm{~d}$ withdrawal period was imposed before conditioned incentive testing to facilitate further development of sensitization.

Microinjection cannula surgery. Rats were anesthetized with ketamine $(80 \mathrm{mg} / \mathrm{kg})$ and xylazine $(5 \mathrm{mg} / \mathrm{kg})$ and were stereotaxically implanted with bilateral 23 gauge guide cannulas targeted at the shell of the nucleus accumbens (day 40, 41, or 42). A slanted skull position was used, with the incisor bar set at $+5.0 \mathrm{~mm}$, and the coordinates were $3.3 \mathrm{~mm}$ anterior to bregma, $1.0 \mathrm{~mm}$ lateral to the midline, and $5.7 \mathrm{~mm}$ ventral to the skull surface. The cannulas were anchored with skull screws and cranial cement, and wire stylets were used to prevent cannula occlusion.
Drugs and microinjections. Bilateral microinjections of vehicle (sterile isotonic saline) or D-amphetamine sulfate $(5.0 \mu \mathrm{g} / 0.5 \mu \mathrm{l}$ per side; Sigma, St. Louis, MO) were administered into the nucleus accumbens in counterbalanced order over two test sessions spaced $48 \mathrm{hr}$ apart. This $5.0 \mu \mathrm{g}$ amphetamine dose was chosen because Wyvell and Berridge (2000) found it produced robust enhancement of cue-triggered wanting for sucrose reward in normal rats. The microinjection cannulas (28 gauge) extended $2.5 \mathrm{~mm}$ beyond the guide cannulas, and the injections were performed with an infusion pump while the rats were gently held $(30 \mathrm{sec}$ duration). The microinjectors were left in place for an additional $1 \mathrm{~min}$ period to allow for drug diffusion. One vehicle microinjection was given before testing began to habituate the rats to the injection procedure and to minimize tissue damage caused by subsequent test injections.

Final lever retraining and extinction exposure. Before testing (days $45-48$ ), rats were given three additional instrumental training sessions to re-establish lever pressing (VI-45 sec schedule), and then one $30 \mathrm{~min}$ session of extinction lever pressing was given to habituate the rats to extinction conditions. Sensitization did not appear to interact with instrumental retraining. Sensitized rats did not press more for sucrose than control rats during the retraining sessions (sensitized rats, $211.15 \pm 16.85$ presses per session; control rats, $226 \pm 17.13$ ). Similarly, sensitized rats were not more resistant to extinction during the extinction habituation trial (sensitized rats, $67.50 \pm 13.81$ presses on the sucrose-associated lever; control rats, $85.14 \pm 17.53$ ). Conditioned incentive testing began the next day.

Conditioned incentive testing. Rats were given a microinjection of vehicle or amphetamine into the nucleus accumbens and immediately placed into the operant chambers for testing (days 49 and 51; $32.5 \mathrm{~min}$ sessions). Instrumental performance was assessed under extinction conditions, so no sucrose pellets were given at any time during the test. In each session, the CS + and CS - were presented four times (30 sec each) on a fixed time, 4 min schedule (in alternating order beginning with the tone stimulus for all rats to counterbalance $\mathrm{CS}+/ \mathrm{CS}-$ order). Presses on both levers were recorded automatically, as were sucrose cup entries. The computer identified lever presses that occurred during each $30 \mathrm{sec}$ CS+ and $\mathrm{CS}-$ presentation. Pressing during the $30 \mathrm{sec}$ period immediately before each $\mathrm{CS}+/ \mathrm{CS}-$ presentation was recorded as the precue baseline. Pressing in the $30 \mathrm{sec}$ period after the cue ended was also recorded. All other emitted behavior was videotaped by the camera positioned below the transparent chamber floor.

Videoscoring of cue-elicited intrusive behavior. A detailed video analysis was made of rearing, sniffing, orienting, and locomotion behavior emitted throughout the incentive test sessions. Scoring was conducted in slow motion (1/10 actual speed) by an observer blind to sensitization versus control group assignments. Occurrences of each behavior were tallied during each $30 \mathrm{sec} C S+$ and during the $30 \mathrm{sec}$ baseline period immediately preceding the CS+ according to the following criteria. (1) Rearing is defined as raising both forelimbs off of the chamber floor and stretching upward toward the chamber ceiling. A rearing bout was considered to have ended if the two forepaws touched the floor. (2) Investigatory sniffing is defined by emitting sniffing movements of the nostrils and vibrissae with the nose within $1 \mathrm{~cm}$ of the chamber wall or corner. Investigatory approaches and sniffing of the lever and food cup were scored separately (together with physical contact with those objects). (3) Body orientation shifts are defined as a sudden shift in body longitudinal axis of at least $90^{\circ}$ within a $2 \mathrm{sec}$ period (both the forelimbs and head-body axis were required to shift together, so that the rat turned the entire body). (4) Locomotion is defined as at least two full consecutive steps forward (faster than $2 \mathrm{~Hz}$ ), that traversed a distance greater than the rat's body length and crossed at least $2 / 3$ of the diameter of the chamber. Each occurrence of rearing and each body orientation shift were scored. Investigatory sniffing and locomotion were scored in $5 \mathrm{sec}$ bins, so that continuous bouts that lasted $>5 \mathrm{sec}$ were scored as two occurrences.

Confirmation of psychomotor sensitization. One day after conditioned incentive testing was completed, a challenge injection of amphetamine $(1.5 \mathrm{mg} / \mathrm{kg}$, i.p.) was given to half of the subjects from both groups to further verify psychomotor sensitization. Rats were placed into the operant chambers (with the levers removed) and videotaped for $45 \mathrm{~min}$ after the injection. Videotape scoring of stereotypy and locomotor activity was conducted for the final drug challenge session, as well as for the first and last sensitization induction sessions, by two observers blind to sensitization-control group assignments. Videotapes were scored in 1 min sampling bins that were spaced every 5 min using a locomotor activity and stereotypy rating scale similar to MacLennan and Maier 
(1983): 1, asleep; 2, inactive; 3, stationary activity such as grooming; 4, mobile, regular activity; 5 , hyperactivity with rearing; 6 , intermittent stereotypic head and limb movements; 7, faster-paced constant stereotypic movements; 8 , intense stereotypic movements focused within a small zone such as a chamber corner; and 9, dyskinesia.

Histology. Rats were deeply anesthetized with sodium pentobarbital, given a microinjection of ink $(0.5 \mu \mathrm{l}$ per side) for site localization, and transcardially perfused with saline and formaldehyde. Brains were extracted, sliced coronally into $50 \mu \mathrm{m}$ sections, and stained with cresyl violet. Microinjection sites were localized in the accumbens shell for 11 subjects in the sensitized group and 11 subjects in the control group. Five rats were found to have one or both cannula outside of the accumbens shell and were excluded from the statistical analyses. An additional three rats were excluded because of illness or cannula obstruction.

Statistics. The data were transformed into difference scores by subtracting the total number of responses during each baseline precue time period from responses during each cue presentation, to isolate the impact of Pavlovian CS + and CS- cues on instrumental performance (Dickinson et al., 2000). ANOVAs (followed by Bonferroni post hoc tests) were performed to examine the between-subject variable of drug sensitization (sensitized group vs control group) and the within-subject variables of cue type (CS + vs CS - ), cue presence (precue baseline vs during the cue), order of cue presentation within a test session (first, second, third, or fourth), and drug microinjection (vehicle vs $5.0 \mu \mathrm{g}$ of amphetamine).

\section{RESULTS}

\section{Confirmation of drug sensitization}

Confirmation of psychomotor sensitization was obtained twice, once during sensitization induction and again after a drug challenge injection at the end of the experiment. During sensitization induction, greater locomotion and stereotypy were observed in the sensitization group on the last day of systemic amphetamine administration than on the first day (two-way ANOVA; day $\times$ time; main effect of day; $\left.F_{(1,139)}=77.04 ; p<0.001\right)$ (Fig. $1 A$ ). During the amphetamine challenge test conducted after the end of conditioned incentive testing, amphetamine $(1.5 \mathrm{mg} / \mathrm{kg}$, i.p.) produced higher locomotor activity and stereotypy scores in sensitized rats than in control rats (two-way ANOVA; group $\times$ time; main effect of group; $F_{(1,90)}=140.99 ; p<0.001$ ) (Fig. $1 B$ ). Thus, robust psychomotor sensitization was achieved by the systemic amphetamine pretreatment regimen.

\section{Sensitization enhancement of cue-triggered lever pressing while drug-free}

Previous amphetamine treatment (sensitization) dramatically elevated cue-triggered pursuit of sucrose after vehicle microinjections. All rats showed a basic conditioned incentive effect, in that the sucrose CS+ triggered more presses on the sucroseassociated lever than in the preceding baseline period when it was absent (two-way ANOVA; CS $+\times$ group; main effect of $\mathrm{CS}+$ presence; $\left.F_{(1,43)}=10.06 ; p<0.01\right)$. However, sensitization magnified the incentive impact of the sucrose cue, and the $\mathrm{CS}+$ triggered $100 \%$ higher increases in pressing in sensitized rats than in control rats (significant group $\times$ CS type interaction; $F_{(1,43)}=$ 5.17; $p<0.05)$. The sensitization-related increase in pressing for sucrose was cue-specific and only occurred during presentations of the CS+ (Bonferroni; $p<0.02)$ and not during the $\mathrm{CS}-(p=$ 0.64 ; NS) (Fig. 2), nor during baseline pressing in the absence of the CS+ (control rats actually lever pressed slightly more than sensitized rats during baseline precue periods) (Fig. 3).

The CS + sucrose cue reversed the baseline pattern of pressing each time it was presented, causing sensitized rats to suddenly lever press more than control rats for the duration of the cue (Fig. 3 ). When pressing during successive pairs of $\mathrm{CS}+$ versus $\mathrm{CS}-$ cues was compared, the difference between the $\mathrm{CS}+$ and $\mathrm{CS}-$
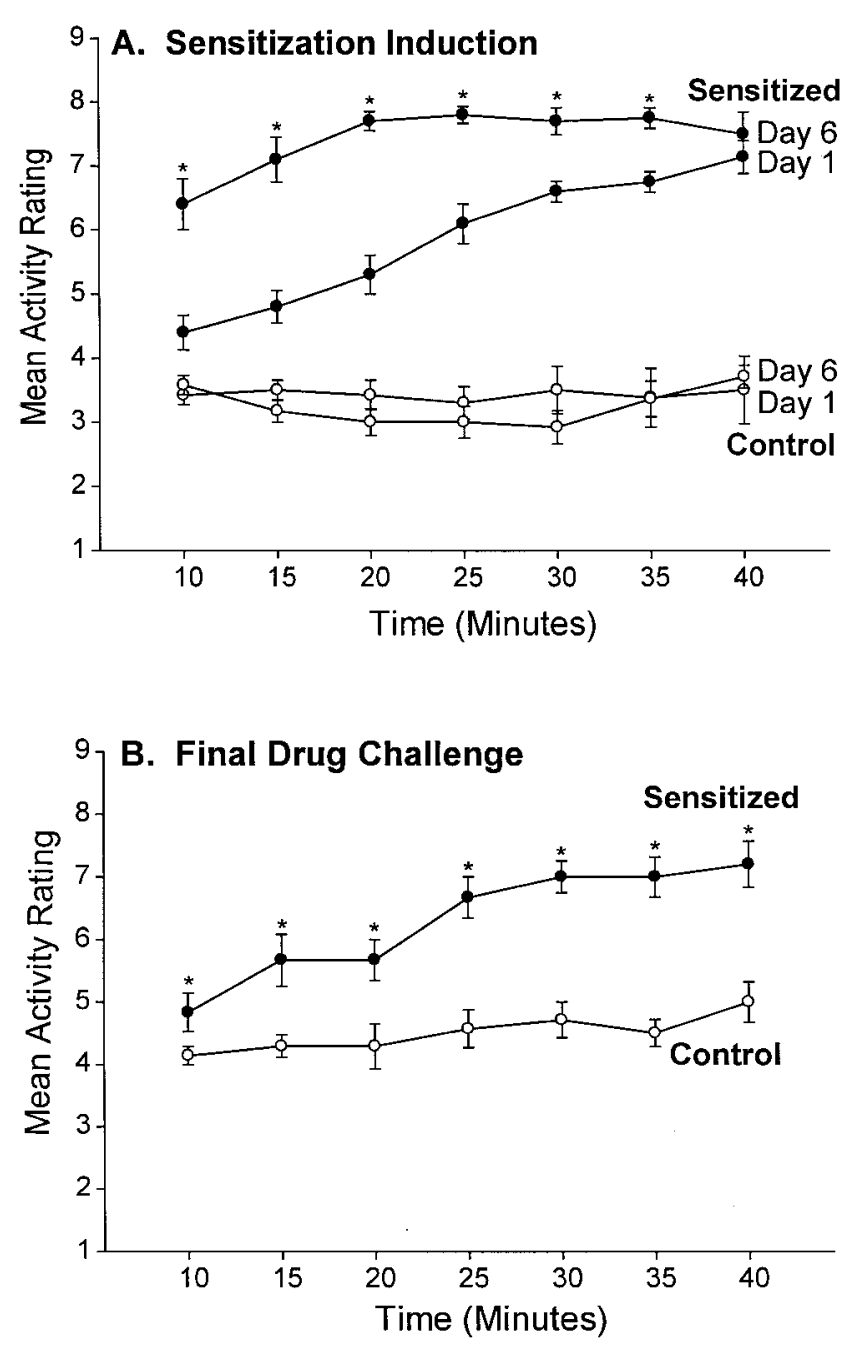

Figure 1. Confirmation of drug sensitization induced by administration of amphetamine. $A$ depicts locomotor and stereotypy ratings during the induction of drug sensitization for the sensitized rats $(3 \mathrm{mg} / \mathrm{kg}$ amphetamine) and control rats $(1 \mathrm{ml} / \mathrm{kg}$ saline). Sensitized rats displayed more locomotor activity and stereotypy on the last day of drug pretreatment compared with the first day, which indicates sensitization to the psychomotor activating effects of amphetamine (two-way ANOVA; ${ }^{*} p<$ 0.01 ; Bonferroni). $B$ depicts locomotor and stereotypy ratings obtained after the completion of conditioned incentive testing, after a systemic challenge injection of amphetamine $(1.5 \mathrm{mg} / \mathrm{kg})$ in both rat groups. The sensitized rats displayed more locomotor activity and stereotypy than the control rats, which further confirms the effectiveness of the drug sensitization regimen (two-way ANOVA; ${ }^{*} p<0.001$; Bonferroni).

was reliably significant only for the sensitized group (sensitized group: two-way ANOVA; CS type $\times$ cue presentation order; main effect of CS type; $\left.F_{(1,87)}=12.53 ; p<0.01\right)$. Sensitized rats emitted more pressing during the $\mathrm{CS}+$ than during the $\mathrm{CS}-$ for each of the first, second, and third presentations of these stimuli pairs within the session (Bonferroni; $p<0.02$ ) (Fig. $4 A$ ). Taken together, this overall pattern of responding indicates that sensitization produces excessive cue-triggered reward "wanting" under drug-free conditions.

\section{Reversibility of sensitized cued-reward pursuit: dependence on cue presence}

Hyperincentive responding by the sensitized rats was temporary, reversible, and repeatable. Intensified sucrose lever pressing was triggered anew by each presentation of the $\mathrm{CS}+$ sucrose cue and 


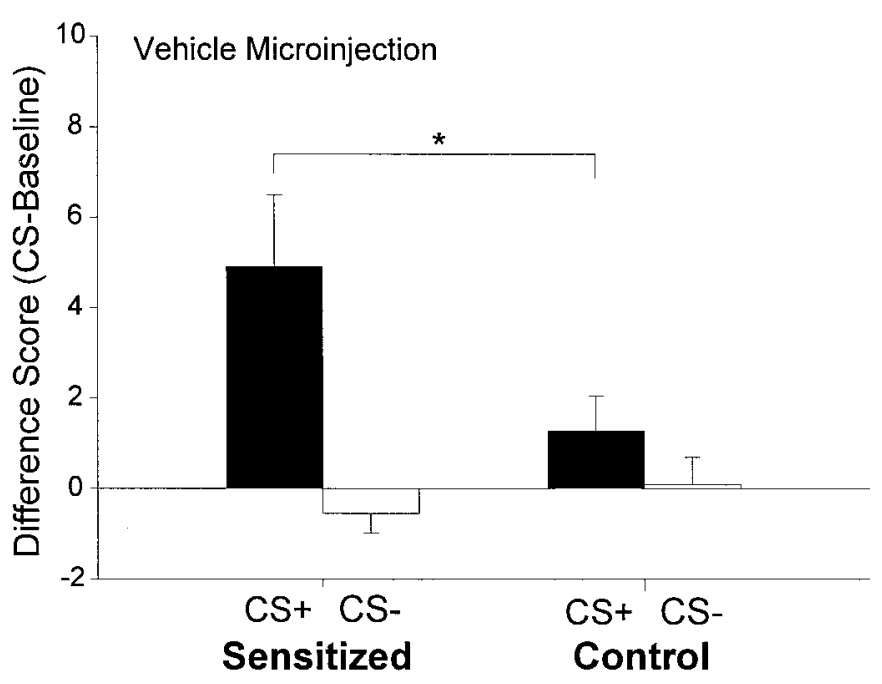

Figure 2. The effect of sensitization on extinction lever pressing for sucrose reward after a microinjection of intra-accumbens vehicle. Sensitized rats engaged in more sucrose $\mathrm{CS}+$ cue-triggered pressing on the sucrose lever than control rats, but sensitized rats did not engage in more pressing during the $\mathrm{CS}-$ cue presentations (two-way ANOVA; ${ }^{*} p<0.02$; Bonferroni).

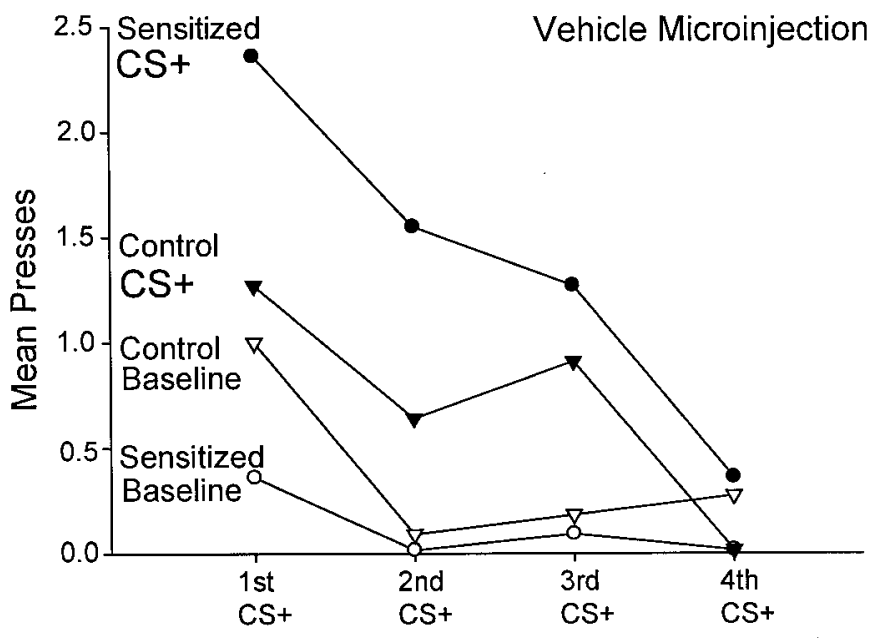

Successive Cue Order in Extinction Test

Figure 3. Absolute responding on the sucrose-associated lever by sensitized and control rats during CS + sucrose cue presentations and baseline precue time periods throughout the extinction test session. Sensitization did not increase baseline lever pressing in the absence of the sucrose cue, because the control rats actually lever pressed slightly more than the sensitized rats during each precue time period. This pattern of responding was reversed by the presence of the cue, however, because the sucrose cue evoked considerably more lever presses from sensitized rats than from control rats during each cue presentation. Thus, the magnitude of the incentive cue effect while drug-free was always greater in sensitized rats than in control rats.

decayed quickly when it ended (sensitized group: two-way ANOVA; cue presence $\times$ presentation order; main effect of cue presence; $F_{(1,87)}=10.68 ; p<0.01$; Bonferroni for first and second cue versus baseline pairs; $p<0.02$ ) (Fig. 3). The reversibility of excessive cue-triggered pursuit in sensitized drug-free rats could be seen when responding was plotted either in terms of difference scores (Fig. $4 A$ ) or in terms of absolute number of presses on the sucrose-associated lever (Fig. $5 A$ ).

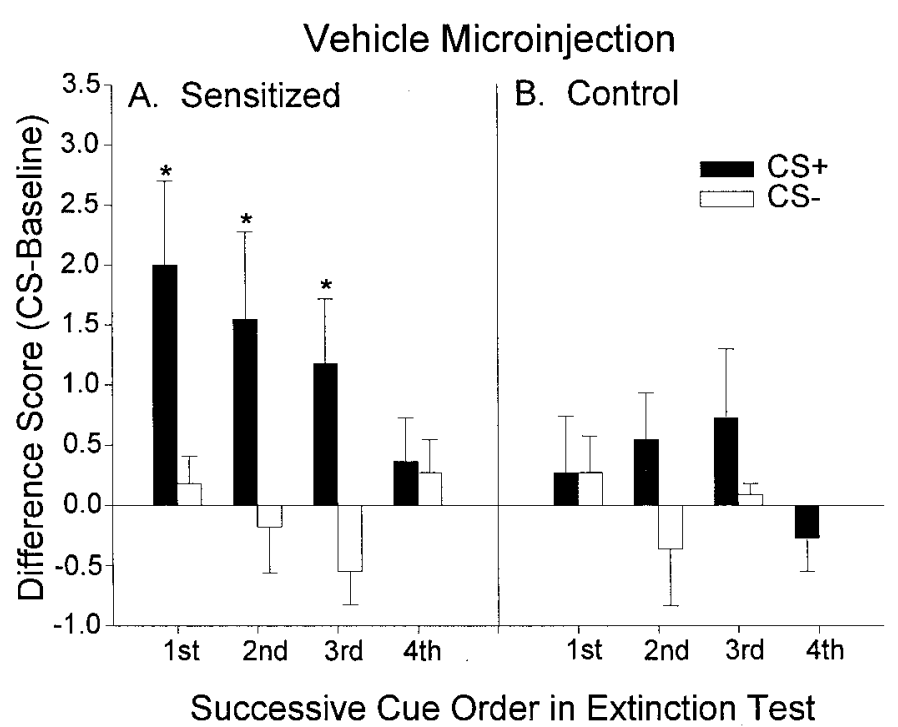

Amphetamine Microinjection

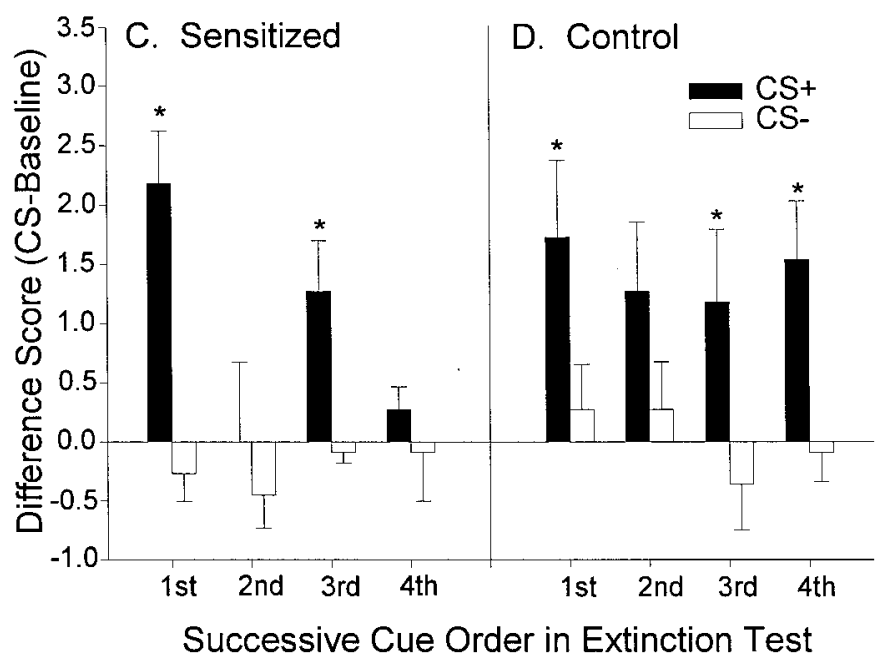

Figure 4. The effect of sensitization and intra-accumbens amphetamine on lever pressing during each successive CS + versus $\mathrm{CS}-$ cue presented over the course of the test sessions. $A$ and $B$ depict lever pressing in the sensitized and control rats after a microinjection of intra-accumbens vehicle. For sensitized rats, more lever pressing was triggered by the sucrose $\mathrm{CS}+$ than by its paired CS - (two-way ANOVA; ${ }^{*} p<0.01$; Bonferroni), and there was a tendency for the sucrose CS + to evoke more presses in the control rats, but this difference was not statistically significant. $C$ and $D$ depict lever pressing in both rat groups after a microinjection of intra-accumbens amphetamine. Intra-accumbens amphetamine boosted sucrose cue-elicited lever pressing in control rats to a level that was comparable with the cue-elicited performance of drug-free sensitized rats, and under the amphetamine microinjection both rat groups engaged in significantly more pressing elicited by the sucrose CS + than by its paired CS - (two-way ANOVAs; ${ }^{*} p<0.05$; Bonferroni).

\section{Effect of intra-accumbens amphetamine on conditioned incentive impact: cue-triggered potentiation in control rats}

For control rats, intra-accumbens amphetamine magnified the cue-triggered increase in pressing on the sucrose lever (two-way ANOVA; microinjection $\times \mathrm{CS}$ type interaction; $F_{(1,43)}=5.36$; $p<0.05)$. The amphetamine microinjection enhanced responding during the $\mathrm{CS}+($ Bonferroni; $p<0.02$ ) but not during the 

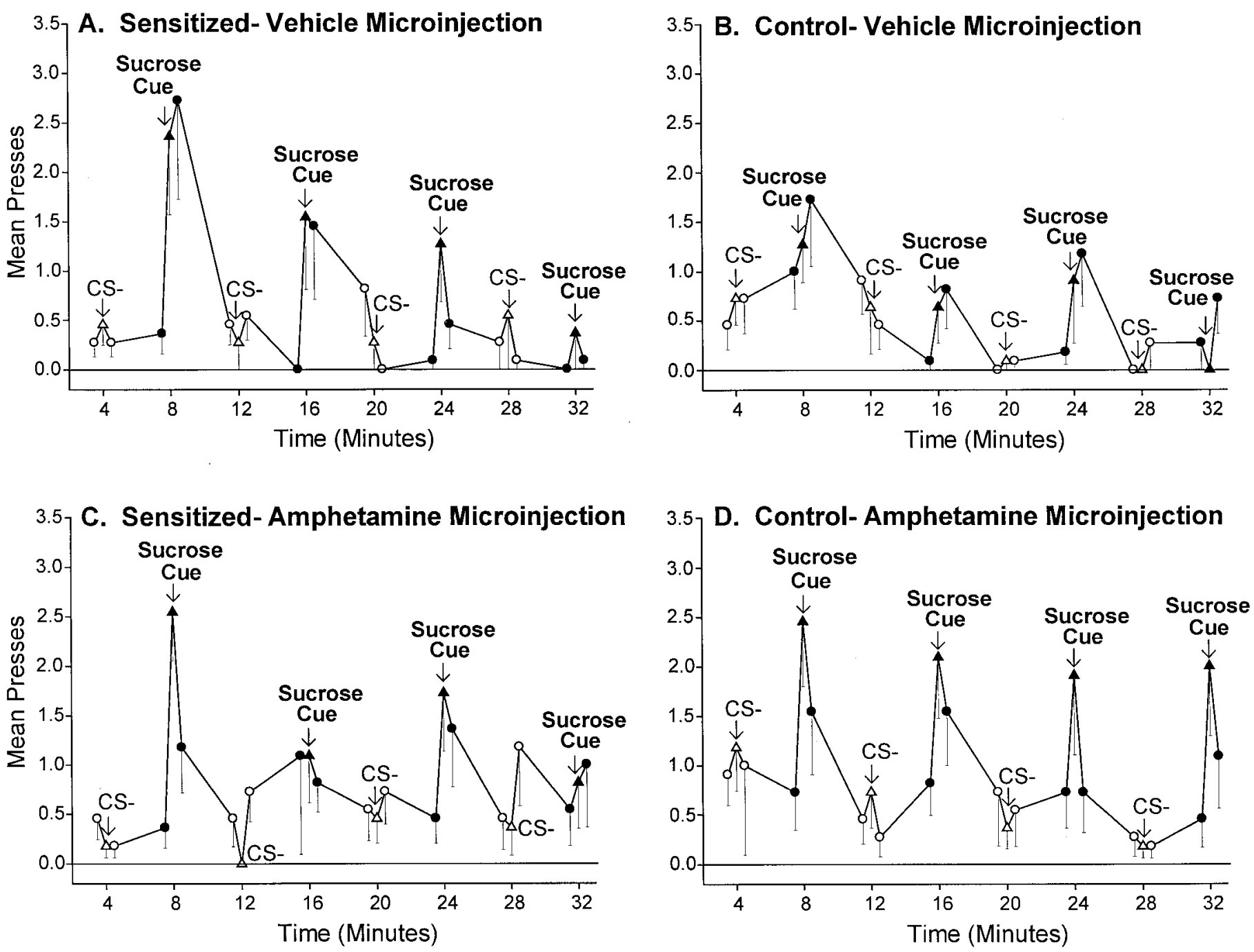

Figure 5. The temporal pattern of the incentive cue effect in sensitized and control rats. The sensitization of cue-elicited pressing was transient and repeatable; sensitized lever pressing was triggered by each presentation of the sucrose cue, but then was followed by a rapid descent back to normal baseline levels of pressing once the cue ended $(A, B)$. Thus, sensitization primarily enhanced "wanting" for sucrose reward when the sucrose cue was actively perceived. Intra-accumbens amphetamine also produced a strikingly similar temporal pattern of effects on the incentive impact of the sucrose cue in both sensitized and control rats $(C, D)$.

CS - and not during baseline. Thus, amphetamine microinjections selectively increased CS + cue-triggered pressing for sucrose reward by normal rats under extinction conditions, consistent with our previous report (Wyvell and Berridge, 2000). The potentiation of cue-triggered "wanting" by amphetamine microinjections in control rats was also apparent in a comparison of each successive pair of CS + versus CS- cues (two-way ANOVA; CS type $\times$ cue presentation order; main effect of CS type; $F_{(1,87)}=$ 17.60; $p<0.01$; Bonferroni tests for first, third, and fourth presentations; $p<0.05$ ) (Fig. 4D).

The enhancement of cue-triggered pressing by amphetamine microinjections in control rats decayed within 1 min after each $\mathrm{CS}+$, only to be repeated when the next $\mathrm{CS}+$ was presented again (Fig. 5D). Thus for control rats, amphetamine microinjections magnified the conditioned incentive impact of the sucrose cue in a manner similar to the sensitization effect described above.

\section{Nonreward control lever comparison}

Few responses were ever made on the second control lever, which had never been associated with sucrose reward, and which served primarily as a measure of general motor arousal (control baseline $=6.5 \pm 2.57$ presses per session after vehicle microinjection; sensitized baseline $=7.73 \pm 2.01$; NS). Pressing on the control lever was not significantly elevated by intra-accumbens amphetamine (control group $=13.91 \pm 5.84$; sensitized group $=10.6 \pm$ 2.41; NS). This is consistent with the finding of Wyvell and Berridge (2000) that amphetamine microinjections increased pressing on the control lever only after a $20 \mu \mathrm{g}$ amphetamine dose and not after the $5 \mu \mathrm{g}$ dose used here. Presentation of the CS+ had no significant effect on pressing on the nonreward lever for either group (three-way ANOVA; NS).

\section{Sensitization interaction with intra-accumbens amphetamine: cue-triggered potentiation versus intrusion}

Sensitized rats showed a robust cue-triggered enhancement of sucrose pursuit after the amphetamine microinjection, just as they had after the vehicle microinjection (two-way ANOVA; CS type $\times$ cue presentation order; main effect of CS type; $F_{(1,87)}=$ 17.32; $p<0.01$ ) (Fig. 4C). For sensitized rats, however, the amphetamine microinjection $(5.0 \mu \mathrm{g})$ did not further enhance 
cue-elicited lever pressing above their already high vehicle levels of cue-triggered pressing (two-way ANOVA; NS). Under amphetamine, the magnitude of cue-triggered pressing by the sensitized and control groups became similar (two-way ANOVA; group $\times$ CS type; no main effects or interaction). In other words, intra-accumbens amphetamine raised the cue-triggered "wanting" effect of the control group to equal the "wanting" effect of the sensitized group but did not further push sensitized lever pressing to an even higher level.

However, the amphetamine microinjection did have a unique effect on the sensitized rats' response to the $\mathrm{CS}+$. This was first detected as a change in cue-triggered lever pressing over the four successive cue presentations within the session, which only occurred for the sensitized group (main effect of CS presentation order, $F_{(3,87)}=3.64, p<0.05$; significant CS type $\times$ CS presentation order interaction, $F_{(3,87)}=2.89, p=0.052$ ) (Fig. $4 C$ ). Specifically, the sucrose CS + triggered more lever pressing than the $\mathrm{CS}-$ during the first and third pairs of cue presentations (Bonferroni, $p<0.05$ ). Yet during the second CS + presentation, the lever pressing of the sensitized group after the amphetamine microinjection was actually lower than their corresponding response after the vehicle microinjection. This led us to examine the possibility that sensitization changed the way that amphetamine modulates behavior elicited by successive reward cues, using the fine-grained video analysis of spontaneous natural behavior recorded during incentive testing.

\section{Sensitization of cue-triggered intrusive behaviors after amphetamine microinjection}

Videoanalysis of spontaneous behaviors emitted during the incentive tests showed that presentations of the CS+ sucrose cue elicited additional behaviors such as rearing, sniffing, orientation shifts, and locomotion from both groups $\left(F_{(1,41)}=29.81 ; p<\right.$ 0.001). This is consistent with reports by Holland (1977) and others that a CS + for reward elicits natural approach conditioned responses (CRs) such as rearing and orientation to the CS+. Under the vehicle microinjection, these natural CRs were not so intense as to compete with lever pressing (regression ANOVA lever pressing versus cue-elicited behaviors emitted by all rats) $\left(F_{(1,83)}=0.50 ; p=0.48\right)$ (Fig. 6A).

The amphetamine microinjection increased the overall emission of intrusive behaviors, such as rearing, sniffing, orientation shifts, and locomotion, by $>500 \%$ even during baseline for both groups $\left(F_{(1,41)}=43.94 ; p<0.001\right)$. Higher numbers of intrusive behaviors under intra-accumbens amphetamine seemed to actually disrupt the performance of lever pressing during the sucrose cue, as revealed by a significant negative correlation between cue-elicited lever pressing and cue-elicited intrusive behavior, which existed for both groups only in the amphetamine condition (Pearson product moment correlation, $r=-0.351, p<0.005$; regression ANOVA, $\left.F_{(1,83)}=11.53, p<0.005\right)($ Fig. $6 B)$. The negative correlation between lever pressing and natural CRs existed only after microinjections of amphetamine, and not after vehicle. This indicates that amphetamine enhanced the expression of CRs, so that they began to intrude and actually compete with lever pressing.

Cues tended to elicit even more intrusive CRs in sensitized rats than in control rats after amphetamine microinjections (two-way ANOVA; group $\times$ type of intrusive behavior during $\mathrm{CS}+$; marginal effect of group $\left.F_{(1,83)}=3.31, p=0.073\right)$. Sensitized cuetriggered intrusive CRs were significantly greater than control CRs during the second CS + presentation $\left(F_{(1,20)}=5.49 ; p<\right.$
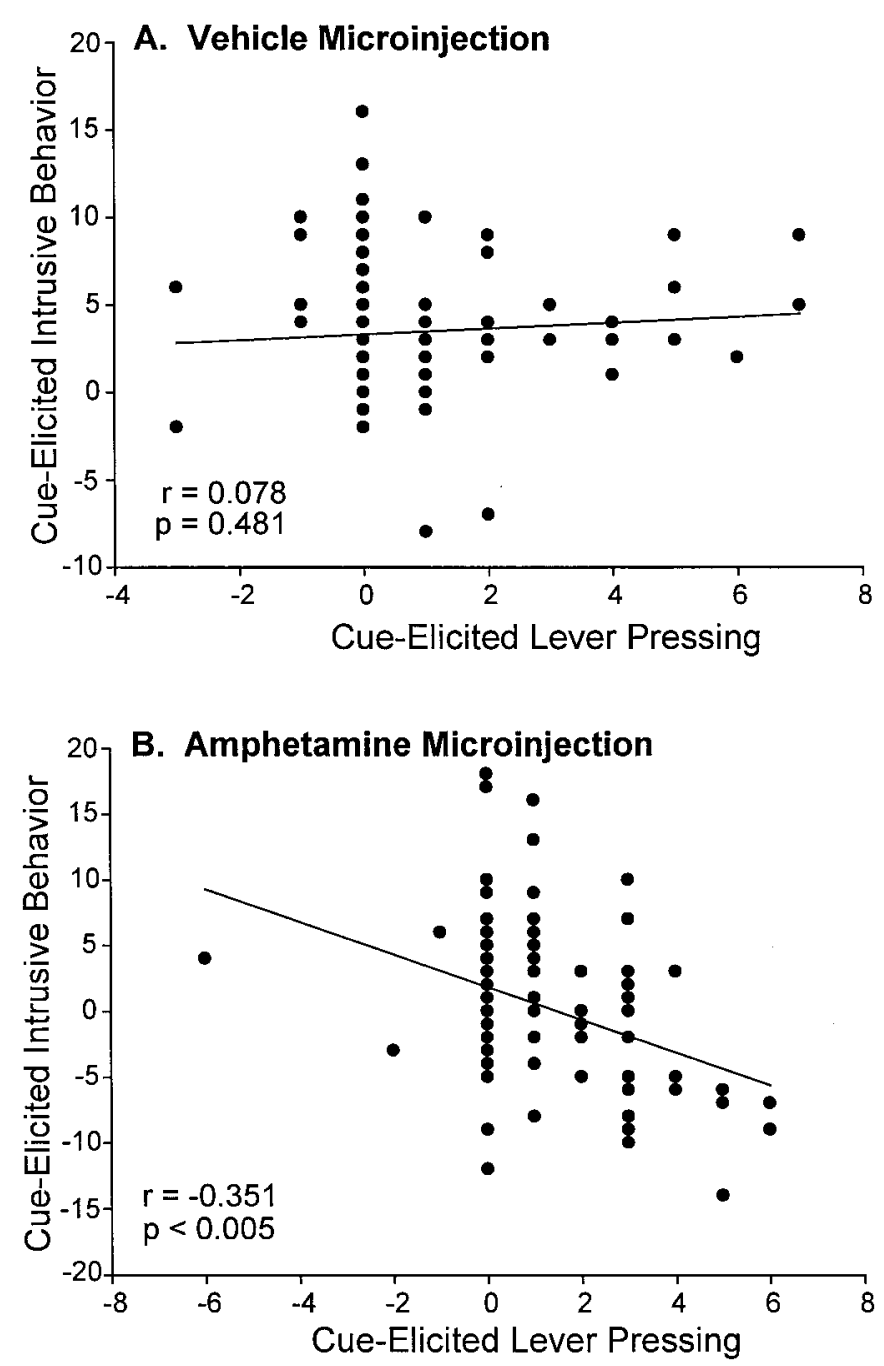

Figure 6. The overall correlation between cue-elicited intrusive behavior and cue-elicited lever pressing in sensitized and control rats. After a microinjection of intra-accumbens amphetamine, there was a significant negative correlation between intrusive behavior and lever pressing during the sucrose cue presentations (Pearson correlation) (B). After the vehicle microinjection, however, there was no significant relationship between cue-elicited intrusive behavior and cue-elicited lever pressing $(A)$, which may be attributable to the lower amount of total intrusive behavior that was expressed under the vehicle testing condition.

0.05) (Fig. 7A), when sensitized cue-triggered lever pressing was lowest. Sensitization potentiated intrusive behaviors only when the $\mathrm{CS}+$ was present and not during the baseline period when it was absent (two-way ANOVA; group $\times$ cue presence; significant interaction $F_{(1,41)}=5.49 ; p<0.05$; Bonferroni test of group during $\mathrm{CS}+; p<0.05)$.

A more fine-grained analysis of intrusive behavior showed that two specific CRs were triggered by the CS + nearly twice as often in sensitized rats than in control rats after amphetamine microinjections, namely rearing and investigatory sniffing behaviors (Bonferroni for sniffing; $p<0.005$ ) (Fig. 7B). Cue-triggered sniffing behavior consisted of leaning forward toward an object in a directed manner (e.g., wall, corner of chamber) while making rhythmic nostril and vibrissae movements. Cue-triggered rearing consisted of standing upwards on the hind legs, while making lateral scanning movements with the head as if searching, often accompanied by sniffing movements too. Sensitized rats also ap- 


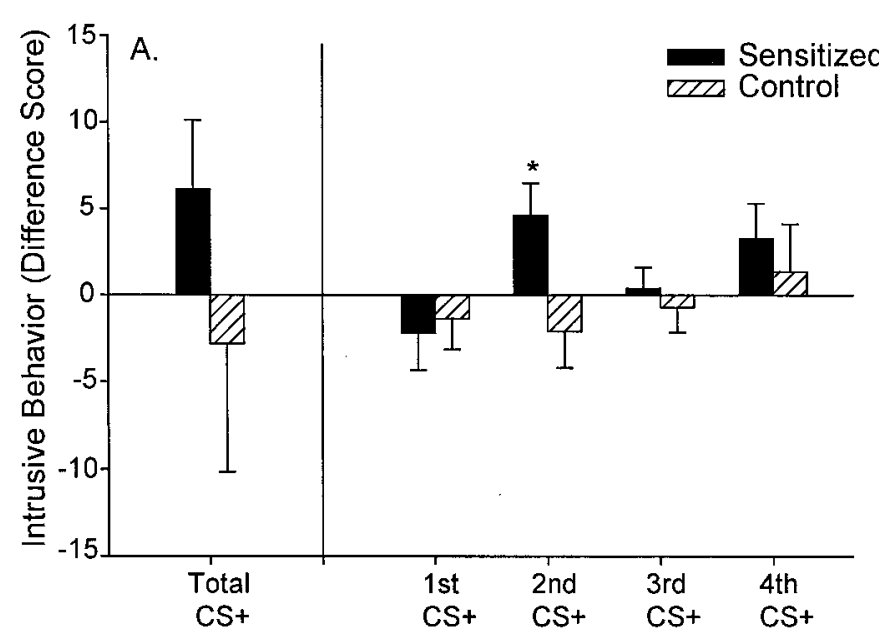

Sucrose Cue Presentations

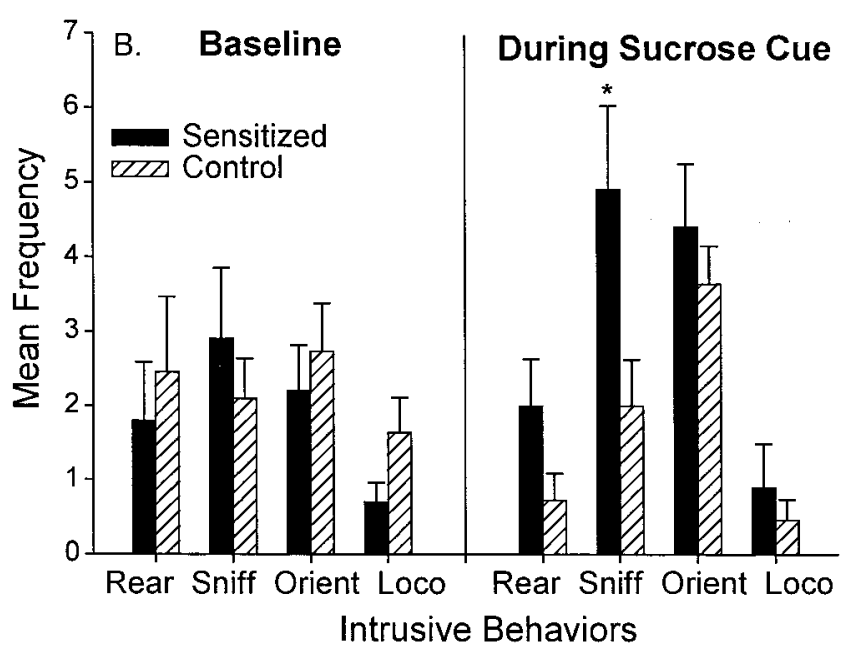

Figure 7. The effect of sensitization on cue-elicited intrusive behaviors. Sensitization tended to increase the amount of cue-elicited intrusive behavior that was expressed after a microinjection of intra-accumbens amphetamine, and this effect was especially apparent during the second presentation of the sucrose cue $(A)$ (one-way ANOVA; ${ }^{*} p<0.05$ ). $B$ shows the frequency of each intrusive behavior emitted during the second sucrose cue in sensitized versus control rats (intrusive behaviors include rearing, sniffing, orientation shifts, and locomotion). Sensitization enhanced intrusive behaviors during the sucrose cue (two-way ANOVA; $p<0.01$; Bonferroni tests for each behavior; $\left.{ }^{*} p<0.005\right)$, but sensitization did not enhance precue baseline intrusive behaviors.

peared to have slight elevations in cue-triggered locomotion and body orientation shifts during the $\mathrm{CS}+$, although those CRs did not differ statistically from control rats (Fig. 7B). Sensitization only increased cue-triggered intrusive behaviors and did not increase baseline emission of these same behaviors in the absence of the $\mathrm{CS}+$.

\section{Histology}

Microinjection sites identified by ink placements are depicted in Figure 8. Drug microinjection sites were restricted to the medial shell of the nucleus accumbens.

\section{DISCUSSION}

Sensitization by amphetamine pre-exposure selectively increased cue-triggered pursuit of sucrose reward, even when rats were

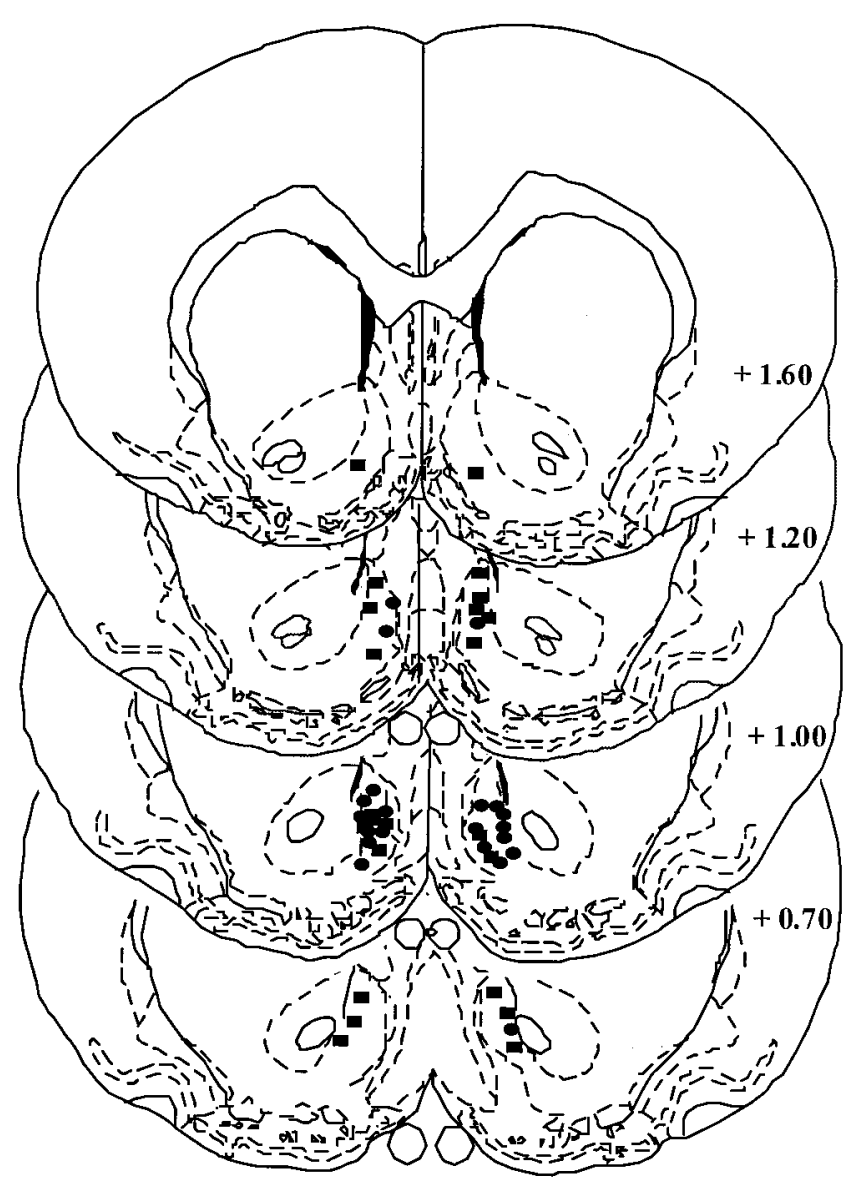

Figure 8. A depiction of the drug microinjection sites localized within the medial shell of the nucleus accumbens. Ovals depict placements from sensitized rats, and rectangles depict placements from control rats. The drawing was adapted from Paxinos and Watson (1986), and the numbers denote distance in millimeters from bregma.

tested while drug-free. The Pavlovian $\mathrm{CS}+$ for sucrose triggered $100 \%$ higher bursts of pressing on the sucrose lever from sensitized rats than from control rats after a vehicle microinjection. Sensitization did not affect pressing during the CS- or during baseline or pressing on the control lever.

This suggests sensitization endowed Pavlovian sucrose cues with hyperincentive properties, causing cues to trigger excessive pursuit of sucrose. These results are consistent with the incentivesensitization theory of addiction, which posits drug-induced sensitization to cause excessive attribution of incentive salience to reward-related cues (Robinson and Berridge, 1993, 2000). Incentive salience is not by itself a normal hunger state, involving deprivation signals or hedonic alliesthesia, nor is it a withdrawal state. Instead incentive salience is posited to be one component of normal appetite, which if attributed to neural representations of reward cues causes both the cues and associated Pavlovian rewards themselves to become attractive and "wanted," triggering approach and pursuit behaviors (Berridge and Valenstein, 1991; Berridge and Robinson, 1998; Berridge, 2001). Our results show that excessive cue-triggered "wanting" persists for at least $10 \mathrm{~d}$ after sensitization induction in the absence of further drug administration. This sensitization effect mimics the excessive cuetriggered reward pursuit caused in normal rats by an amphetamine microinjection into the nucleus accumbens (Wyvell and Berridge, 2000). 


\section{Perceptual gating of incentive sensitization: reversible and repeated reward wanting}

Excessive pursuit of sucrose by sensitized rats was gated by actual perception of the CS+ sucrose cue, just as after intra-accumbens amphetamine in normal rats (Wyvell and Berridge, 2000). For sensitized drug-free rats, and for all rats after amphetamine microinjections, bursts of pressing on the sucrose lever came and went with the presence of the $30 \mathrm{sec}$ auditory $\mathrm{CS}+$. Increased pressing was triggered as soon as the sucrose cue began but declined sharply back to normal baseline levels when the cue was turned off.

This on-off pattern of pressing bursts can be understood if sensitization specifically magnified the attribution of incentive salience to the CS+ (Berridge and Robinson, 1998). However, once the hyperincentive cue was no longer present, motivation for sucrose reward would instead be guided primarily by other processes, ranging from simple associative response habits to cognitive act-outcome expectations, mediated by separate neural systems (Balleine and Killcross, 1994; Dickinson and Balleine, 1994; Balleine and Dickinson, 1998, 2001; Dickinson et al., 2000; Berridge, 2001).

\section{Alternative interpretations of the sensitized incentive cue effect}

Sensitization has been reported to increase other reward measures, such as place preference, conditioned reinforcement, and instrumental break point (Lett, 1989; Cunningham and Kelley, 1992; Shippenberg and Heidbreder, 1995; Harmer and Phillips, 1998; Pierre and Vezina, 1998; Deroche et al., 1999; Taylor and Horger, 1999; Lorrain et al., 2000; Marinelli and White, 2000). Although such increases might be explained by incentivesensitization, a number of other explanations are also possible, such as sensitization enhancement of conditioned reinforcement, associative reward learning, or even primary reinforcement (when reward is actually obtained). Our use of a conditioned incentive paradigm excluded these alternative explanations of cue-triggered "wanting," as described below.

\section{Conditioned response reinforcement}

Sensitization could not have acted by increasing conditioned response reinforcement, although conditioned reinforcement may be increased by sensitization (Cunningham and Kelley, 1992; Kelley and Holahan, 1997; Taylor and Horger, 1999). The Pavlovian reward cue was never contingent on lever pressing, but instead was presented freely regardless of whether the rats pressed the lever. The cue elicited subsequent pressing, rather than reinforced previous responses.

\section{Conditioned Pavlovian $S-R$ habit}

Sensitization could not have enhanced pressing by strengthening behavioral habits elicited by the $\mathrm{CS}+$. The cue had never before been paired with the act of lever pressing, so lever pressing was not a cue-triggered habit. In fact, sensitized conditioned responses elicited by the CS + tended to compete with lever pressing after intra-accumbens amphetamine, rather than to facilitate pressing.

\section{Hedonic impact of primary reward or primary response reinforcement}

Sensitization could not have increased reward pursuit by enhancing the hedonic impact of sucrose reward, because sucrose was never delivered during the extinction test. There was no primary response reinforcement during testing, and therefore sensitization could not have acted through it.

\section{Formation of Pavlovian associations}

Sensitization could not have acted by facilitating stimulus-reward learning, although mesolimbic dopamine may modulate the ability of CSs to enter into diverse Pavlovian associations. For example, sensitization increases the formation of Pavlovian inhibitory associations to a sucrose $\mathrm{CS}-$, as well as excitatory associations to a CS+ (Harmer and Phillips, 1999b), and systemic or intraaccumbens dopamine agonists can overcome the usual latent inhibition of associability of a CS + normally caused by its preexposure before training (Killcross et al., 1994; Gray et al., 1997; Joseph et al., 2000). Such findings highlight motivational-attentional-associative interactions in mesolimbic function. Based on such association interactions, several authors have suggested that the role of sensitization in addiction is chiefly to facilitate stimulus-reward learning (Di Chiara, 1999; Robbins and Everitt, 1999; Berke and Hyman, 2000). However, it is important to note that in our experiment all Pavlovian conditioning occurred before sensitization, and therefore our sensitized cue-triggered effects were not attributable to enhanced formation of Pavlovian associations. Instead, sensitization was restricted to magnifying the incentive value of the CS+ that had already been gained based on previously formed Pavlovian associations.

\section{Strength of existing reward association}

Sensitization is unlikely to have increased cue-triggered pursuit by enhancing the general strength of existing reward associations. There were many other reward-associated stimuli in the chamber besides the sucrose cue even during baseline periods (i.e., sucrose lever, sucrose cup, etc), but sensitization did not enhance the ability of those cues to increase baseline pressing (probably because their constant presence diluted their Pavlovian correlation to reward). Thus, the increased motivation was unlikely to be attributable to enhancement of the strength of reward prediction or general associations.

Instead, the sensitization increase was tied to the $\mathrm{CS}+$ that had been associatively paired with sucrose in an explicit Pavlovian manner, as predicted by the incentive-sensitization theory (Robinson and Berridge, 1993, 2000). Although we recognize mesoaccumbens systems to have additional complex sensorimotor and other functions (Salamone et al., 1997; Everitt et al., 1999; Horvitz, 2000), it is difficult to explain our results based on those hypotheses. The best interpretation of our results seems to be that sensitization caused reward cues to trigger increased incentive salience or "wanting" for the associated sucrose reward (just as drug cues are posited to trigger addicts to "want" associated drug rewards).

\section{Impact of intra-accumbens amphetamine on sensitized cue-triggered incentive salience}

Although it was at first surprising that amphetamine microinjections failed to further potentiate the lever pressing of sensitized rats above their already high vehicle levels, this was explained once it became clear that sensitization also increased the competitive disruption by intrusive behaviors that occurs under intraaccumbens amphetamine (e.g., investigatory sniffing, rearing). Intrusive behaviors were magnified versions of Pavlovian conditioned responses such as approach, orienting, rearing, and freezing that are normally elicited by a reward CS+ (Holland, 1977). Cue-triggered intrusive behaviors were facilitated in all rats by 
amphetamine microinjections, but sensitized rats emitted the greatest number of intrusions.

One possible mechanism for increased intrusive behaviors might be a synergistic elevation of incentive salience, caused by combination of sensitization and amphetamine microinjection, which disrupted prefrontal coordination of goal-directed lever pressing (Seamans et al., 1995; Balleine and Dickinson, 1998). The prefrontal cortex may ordinarily modulate cue-triggered activation in accumbens (Schultz, 1998; Ciccocioppo et al., 2001; Jackson and Moghaddam, 2001) and prevent excessive behavioral responses. Prefrontal dysfunction in addiction has been argued to disrupt such prefrontal modulation of the accumbens systems (Jentsch and Taylor, 1999; Volkow and Fowler, 2000; Bechara et al., 2001; Robinson et al., 2001), and disruptive orientation and approach to excessively "wanted" cues might interfere with goaldirected strategies of lever pressing. Although our sensitization regimen was probably too mild to produce significant prefrontal dysfunction, it seems possible that the combined effects of amphetamine microinjection and sensitization were sufficient to overcome normal modulation of the accumbens response to cues in decision making. Thus, goal-directed strategies of reward pursuit may have been partially disrupted in sensitized rats by excessive cue-triggered orienting responses.

\section{Focus of excessive "wanting:" natural rewards versus drug rewards}

By using a natural reward, sucrose, our results isolated excessive cue-triggered incentive salience caused by sensitization from contamination by other processes that would co-occur when using drug rewards, such as withdrawal. Our finding that sensitization caused excessive cue-triggered "wanting" for sucrose is consistent with other reports of sensitized pursuit of natural rewards such as sucrose or sex incentives (Mitchell and Stewart, 1990a; Fiorino and Phillips, 1999a,b). For drug addiction, however, the focus of sensitized "wanting" is posited by the incentive-sensitization theory to be primarily toward drug cues and drug rewards, rather than natural rewards (Robinson and Berridge, 1993, 2000). Future studies will be needed to examine factors that determine whether it is drug rewards or natural rewards that become excessively "wanted" after sensitization, as well as contextual gating factors (Anagnostaras and Robinson, 1996; Robinson et al., 1998; Crombag et al., 2000).

\section{Conclusion}

Encounters with reward cues trigger enhanced incentive salience of their associated rewards, rather than merely reinforcing responses or triggering habitual actions. Sensitization interacts synergistically with reward cues, allowing a cue to trigger hyperincentive "wanting" for the reward. For normal individuals, excessive cue-triggered "wanting" requires mesolimbic activation by an amphetamine microinjection. Previous drug exposure (sensitization), however, causes hyperincentive motivation to be triggered by reward cues even when individuals are in a drug-free state. In addition, sensitization increases generation of cued intrusive responses that may disrupt goal pursuit after intraaccumbens amphetamine. We believe these results are the purest demonstration so far of the incentive-sensitization theory postulate that sensitization enhances the cue-triggered incentive salience of rewards (Robinson and Berridge, 1993, 2000), in an experiment that cannot be explained alternatively in terms such as increased reinforcer impact, associative reward learning, or conditioned reinforcement.

\section{REFERENCES}

Anagnostaras SG, Robinson TE (1996) Sensitization to the psychomotor stimulant effects of amphetamine: modulation by associative learning. Behav Neurosci 110:1397-1414.

Balleine B, Killcross S (1994) Effects of ibotenic acid lesions of the nucleus accumbens on instrumental action. Behav Brain Res 65:181-193.

Balleine BW, Dickinson A (1998) Goal-directed instrumental action: contingency and incentive learning and their cortical substrates. Neuropharmacology 37:407-419.

Bechara A, Dolan S, Denburg N, Hindes A, Anderson SW, Nathan PE (2001) Decision-making deficits, linked to a dysfunctional ventromedial prefrontal cortex, revealed in alcohol and stimulant abusers. Neuropsychologia 39:376-389.

Berke JD, Hyman SE (2000) Addiction, dopamine, and the molecular mechanisms of memory. Neuron 25:515-532.

Berridge KC (2001) Reward learning: reinforcement, incentives, and expectations. In: The psychology of learning and motivation (Medin DL, ed), pp 223-278. New York: Academic.

Berridge KC, Robinson TE (1998) What is the role of dopamine in reward: hedonic impact, reward learning, or incentive salience? Brain Res Brain Res Rev 28:309-369.

Berridge KC, Valenstein ES (1991) What psychological process mediates feeding evoked by electrical stimulation of the lateral hypothalamus? Behav Neurosci 105:3-14.

Ciccocioppo R, Sanna PP, Weiss F (2001) Cocaine-predictive stimulus induces drug-seeking behavior and neural activation in limbic brain regions after multiple months of abstinence: reversal by $\mathrm{D}(1)$ antagonists. Proc Natl Acad Sci USA 98:1976-1981.

Crombag HS, Badiani A, Maren S, Robinson TE (2000) The role of contextual versus discrete drug-associated cues in promoting the induction of psychomotor sensitization to intravenous amphetamine. Behav Brain Res 116:1-22.

Cunningham ST, Kelley AE (1992) Evidence for opiate-dopamine crosssensitization in nucleus accumbens: studies of conditioned reward. Brain Res Bull 29:675-680.

Deroche V, Le Moal M, Piazza PV (1999) Cocaine self-administration increases the incentive motivational properties of the drug in rats. Eur J Neurosci 11:2731-2736.

de Wit H, Stewart J (1981) Reinstatement of cocaine-reinforced responding in the rat. Psychopharmacology 75:134-143.

Di Chiara G (1998) A motivational learning hypothesis of the role of mesolimbic dopamine in compulsive drug use. J Psychopharmacol 12:54-67.

Di Chiara G (1999) Drug addiction as dopamine-dependent associative learning disorder. Eur J Pharmacol 375:13-30.

Dickinson A, Balleine B (1994) Motivational control of goal-directed action. Anim Learn Behav 22:1-18.

Dickinson A, Smith J, Mirenowicz J (2000) Dissociation of Pavlovian and instrumental incentive learning under dopamine antagonists. Behav Neurosci 114:468-483.

Dickinson AJ, Balleine B (2001) The role of learning in motivation. In: Steven's handbook of experimental psychology, Ed 3. New York: Wiley, in press.

Everitt BJ, Parkinson JA, Olmstead MC, Arroyo M, Robledo P, Robbins TW (1999) Associative processes in addiction and reward: the role of amygdala-ventral striatal subsystems. Ann NY Acad Sci 877:412-438.

Fiorino DF, Phillips AG (1999a) Facilitation of sexual behavior and enhanced dopamine efflux in the nucleus accumbens of male rats after D-amphetamine-induced behavioral sensitization. J Neurosci 19:456-463.

Fiorino DF, Phillips AG (1999b) Facilitation of sexual behavior in male rats following D-amphetamine-induced behavioral sensitization. Psychopharmacology 142:200-208.

Goldstein A (1994) Addiction: from biology to drug policy. New York: Freeman.

Gray JA, Moran PM, Grigoryan G, Peters SL, Young AM, Joseph MH (1997) Latent inhibition: the nucleus accumbens connection revisited. Behav Brain Res 88:27-34.

Harmer CJ, Phillips GD (1998) Enhanced appetitive conditioning following repeated pretreatment with D-amphetamine. Behav Pharmacol 9:299-308.

Harmer CJ, Phillips GD (1999a) Enhanced dopamine efflux in the amygdala by a predictive, but not a non-predictive, stimulus: facilitation by prior repeated D-amphetamine. Neuroscience 90:119-130.

Harmer CJ, Phillips GD (1999b) Enhanced conditioned inhibition following repeated pretreatment with D-amphetamine. Psychopharmacology 142:120-131.

Holland PC (1977) Conditioned stimulus as a determinant of the form of the Pavlovian conditioned response. J Exp Psychol Anim Behav Process 3:77-104.

Horvitz JC (2000) Mesolimbocortical and nigrostriatal dopamine responses to salient non-reward events. Neuroscience 96:651-656.

Ito R, Dalley JW, Howes SR, Robbins TW, Everitt BJ (2000) Dissocia- 
tion in conditioned dopamine release in the nucleus accumbens core and shell in response to cocaine cues and during cocaine-seeking behavior in rats. J Neurosci 20:7489-7495.

Jackson ME, Moghaddam B (2001) Amygdala regulation of nucleus accumbens dopamine output is governed by the prefrontal cortex. J Neurosci 21:676-681.

Jaffe JH, Cascella NG, Kumor KM, Sherer MA (1989) Cocaineinduced cocaine craving. Psychopharmacology 97:59-64.

Jentsch JD, Taylor JR (1999) Impulsivity resulting from frontostriatal dysfunction in drug abuse: implications for the control of behavior by reward-related stimuli. Psychopharmacology 146:373-390.

Joseph MH, Peters SL, Moran PM, Grigoryan GA, Young AMJ, Gray JA (2000) Modulation of latent inhibition in the rat by altered dopamine transmission in the nucleus accumbens at the time of conditioning. Neuroscience 101:921-930.

Kelley AE, Holahan MR (1997) Enhanced reward-related responding following cholera toxin infusion into the nucleus accumbens. Synapse 26:46-54

Killcross AS, Dickinson A, Robbins TW (1994) Amphetamine-induced disruptions of latent inhibition are reinforcer mediated: implications for animal models of schizophrenic attentional dysfunction. Psychopharmacology 115:185-195.

Koob GF, Le Moal M (2001) Drug addiction, dysregulation of reward, and allostasis. Neuropsychopharmacology 24:97-129.

Lett BT (1989) Repeated exposures intensify rather than diminish the rewarding effects of amphetamine, morphine, and cocaine. Psychopharmacology 98:357-362.

Lorrain DS, Arnold GM, Vezina P (2000) Previous exposure to amphetamine increases incentive to obtain the drug: long-lasting effects revealed by the progressive ratio schedule. Behav Brain Res 107:9-19.

MacLennan AJ, Maier SF (1983) Coping and the stress-induced potentiation of stimulant stereotypy in the rat. Science 219:1091-1093.

Marinelli M, White FJ (2000) Enhanced vulnerability to cocaine selfadministration is associated with elevated impulse activity of midbrain dopamine neurons. J Neurosci 20:8876-8885.

Mitchell JB, Stewart J (1990a) Facilitation of sexual behaviors in the male rat in the presence of stimuli previously paired with systemic injections of morphine. Pharmacol Biochem Behav 35:367-372.

Mitchell JB, Stewart J (1990b) Facilitation of sexual behaviors in the male rat associated with intra-VTA injections of opiates. Pharmacol Biochem Behav 35:643-650.

Mucha RF, Geier A, Pauli P (1999) Modulation of craving by cues having differential overlap with pharmacological effect: evidence for cue approach in smokers and social drinkers. Psychopharmacology 147:306-313.

Paxinos G, Watson C (1986) The rat brain in stereotaxic coordinates, Ed 2. Sydney: Academic.
Pierre PJ, Vezina P (1998) D-1 dopamine receptor blockade prevents the facilitation of amphetamine self administration induced by prior exposure to the drug. Psychopharmacology 138:159-166.

Robbins SJ, Ehrman RN, Childress AR, O'Brien CP (1999) Comparing levels of cocaine cue reactivity in male and female outpatients. Drug Alcohol Depend 53:223-230.

Robbins TW, Everitt BJ (1999) Drug addiction: bad habits add up. Nature 398:567-570.

Robinson TE, Berridge KC (1993) The neural basis of drug craving: an incentive-sensitization theory of addiction. Brain Res Brain Res Rev 18:247-291.

Robinson TE, Berridge KC (2000) The psychology and neurobiology of addiction: an incentive-sensitization view. Addiction 95:91-117.

Robinson TE, Browman KE, Crombag HS, Badiani A (1998) Modulation of the induction or expression of psychostimulant sensitization by the circumstances surrounding drug administration. Neurosci Biobehav Rev 22:347-354.

Robinson TE, Gorny G, Mitton E, Kolb B (2001) Cocaine selfadministration alters the morphology of dendrites and dendritic spines in the nucleus accumbens and neocortex. Synapse 39:257-266.

Salamone JD, Cousins MS, Snyder BJ (1997) Behavioral functions of nucleus accumbens dopamine: empirical and conceptual problems with the anhedonia hypothesis. Neurosci Biobehav Rev 21:341-359.

Schultz W (1998) Predictive reward signal of dopamine neurons. J Neurophysiol 80:1-27.

Seamans JK, Floresco SB, Phillips AG (1995) Functional differences between the prelimbic and anterior cingulate regions of the rat prefrontal cortex. Behav Neurosci 109:1063-1073.

Shippenberg TS, Heidbreder C (1995) Sensitization to the conditioned rewarding effects of cocaine: pharmacological and temporal characteristics. J Pharmacol Exp Ther 273:808-815.

Siegel S (1999) Drug anticipation and drug addiction. The 1998 H. David Archibald Lecture. Addiction 94:1113-1124.

Taylor JR, Horger BA (1999) Enhanced responding for conditioned reward produced by intra-accumbens amphetamine is potentiated after cocaine sensitization. Psychopharmacology 142:31-40.

Topp L, Lovibond PF, Mattick RP (1998) Cue reactivity in dependent amphetamine users: can monistic conditioning theories advance our understanding of reactivity? Drug Alcohol Rev 17:277-288.

Volkow ND, Fowler JS (2000) Addiction, a disease of compulsion and drive: involvement of the orbitofrontal cortex. Cereb Cortex $10: 318-325$

Wyvell CL, Berridge KC (2000) Intra-accumbens amphetamine increases the conditioned incentive salience of sucrose reward: enhancement of reward "wanting" without enhanced "liking" or response reinforcement. J Neurosci 20:8122-8130. 